\title{
Efecto de la relación potasio/sodio en la pradera sobre el comportamiento ingestivo de vacas lecheras
}

\author{
Effect of potassium/sodium ratio in the pasture \\ on ingestive behaviour of dairy cows
}

\author{
Herrera, F. ${ }^{a}$, Balocchi, 0. ${ }^{b}$ Riegel, R. ${ }^{c}$, Keim, J.P. ${ }^{b *}$ \\ ${ }^{a}$ Escuela de Graduados, Facultad de Ciencias Agrarias, Universidad Austral de Chile \\ ${ }^{b}$ Instituto de Producción Animal, Facultad de Ciencias Agrarias, Universidad Austral de Chile \\ ${ }^{c}$ Instituto de Producción y Sanidad Vegetal, Facultad de Ciencias Agrarias, Universidad Austral de Chile.
}

\begin{tabular}{l} 
A R T I C L E I N F O \\
\hline Article history: \\
Received 04.07.2016 \\
Accepted 05.12.2016 \\
\hline Keywords: \\
Voluntary intake \\
Potassium/sodium ratio \\
Grazing behavior \\
\hline Original Research Article, \\
Special Issue: Pastures for \\
Sustainable Productions Systems \\
\hline *Corresponding author: \\
Juan Pablo Keim \\
E-mail address: \\
juan.keim@uach.cl
\end{tabular}

A B S T R A C T

The aim of this study was to determine the effect of changing the potassium/sodium ratio in a Lolium perenne L. sward on ingestive behaviour of lactating dairy cows. The experiment considered $5 \mathrm{~K} / \mathrm{Na}$ ratios that were organised in $10 \times 20 \mathrm{~m}$ plots arranged in three randomized complete blocks. To generate the different K/Na ratios, 0 kg K $\mathrm{O}-0 \mathrm{~kg} \mathrm{Na}$ (control), $50 \mathrm{~kg} \mathrm{Na} \mathrm{ha}^{-1}, 100 \mathrm{~kg} \mathrm{Na} \mathrm{ha}^{-1}$, $400 \mathrm{~kg} \mathrm{~K}_{2} \mathrm{O} \mathrm{ha}{ }^{-1}$ and $800 \mathrm{~kg} \mathrm{~K}_{2} \mathrm{O}$ ha $^{-1}$ were applied to the different treatments. Plots were grazed by lactating dairy cows, which were offered an herbage mass of $6 \mathrm{~kg} \mathrm{DM} \mathrm{cow}^{-1}$ (above $5 \mathrm{cms}$ ground level) during a $6 \mathrm{~h}$ grazing event. Nutritional composition, $\mathrm{K}$ and $\mathrm{Na}$ concentration in the pasture, pasture height and herbage mass, as well as pasture removal and grazing behaviour were assesed. Evaluations were conducted at three different seasons (May, September and October). Data were subjected to anova using the proc MIXED of SAS, with the different K/Na ratios as a fixed effect and grazing season as a repeated measure. Fertiliser levels modified $\mathrm{K}$ and Na concentrations in the pasture, generating K/Na ratios that ranged between 3 and 48 for $100 \mathrm{~kg} \mathrm{Na} \mathrm{ha}^{-1}$ y $800 \mathrm{~kg} \mathrm{~K}_{2} \mathrm{O}$ $h^{-1}$ respectively $(\mathrm{P}<0,05)$. Nevertheless, the $\mathrm{K} / \mathrm{Na}$ ratio in the pasture had no effect on ingestive behaviour, herbage mass and chemical composition of the pasture. It is concluded that under the conditions of this experiment, $\mathrm{K} / \mathrm{Na}$ ratio in the pasture has no effect on grazing behaviour of lactanting dairy cows.

\section{RESUMEN}

El objetivo del presente estudio fue determinar el efecto de la relación potasio/sodio en Lolium perenne sobre el comportamiento ingestivo de vacas lecheras. Se establecieron cinco tratamientos (relación K/Na) en parcelas de 10x20 m organizadas en tres bloques completos al azar. Para generar relaciones K/Na contrastantes se aplicaron $0 \mathrm{~kg} \mathrm{Ky} 0 \mathrm{~kg} \mathrm{Na}$ (testigo), 50 y $100 \mathrm{~kg} \mathrm{Na} \mathrm{ha-1,}$ 400 y $800 \mathrm{~kg} \mathrm{~K}_{2} \mathrm{O} \mathrm{ha}^{-1}$. Las parcelas fueron pastoreadas por vacas lecheras en lactancia, con una oferta de $6 \mathrm{~kg} \mathrm{MS} \mathrm{vaca}^{-1}$ (sobre $5 \mathrm{~cm}$ ), durante un ciclo de pastoreo de 6 horas. Las variables evaluadas fueron composición nutricional y concentración de K y Na en la pradera, fitomasa y altura de la pradera, comportamiento en pastoreo y dinámica de defoliación. Las evaluaciones se realizaron en tres épocas del año (mayo, septiembre y octubre). Los datos fueron sometidos a análisis de varianza mediante el procedimiento MIXED de SAS con las diferentes relaciones K/Na como factor fijo y la época de pastoreo como medida repetida en el tiempo. Las distintas dosis de fertilización modificaron las concentraciones de K y Na en la pradera, generando relaciones $\mathrm{K} / \mathrm{Na}$ contrastantes (3 y 48 para $100 \mathrm{~kg} \mathrm{Na} \mathrm{ha}^{-1}$ y $800 \mathrm{~kg} \mathrm{~K}_{2} \mathrm{O}$ ha-1 respectivamente) $(\mathrm{P}<0,05$ ). No obstante, la relación $\mathrm{K} / \mathrm{Na}$ en la pradera no afectó el consumo voluntario, el rendimiento, ni la calidad nutritiva de la pradera. En conclusión, las diferentes relaciones $\mathrm{K} / \mathrm{Na}$ en praderas de Lolium perenne no afectaron el comportamiento ingestivo de vacas lecheras bajo las condiciones de este estudio.

Palabras clave: consumo voluntario, relación potasio/sodio, comportamiento de pastoreo.

\section{INTRODUCCIÓN}

Los sistemas de producción de leche en base a pastoreo son una alternativa competitiva de producción en zonas de clima templado en la medida que la pradera presente un buen nivel de calidad y rendimiento de materia seca a lo largo del año (Balocchi et al., 2013), ya que producir $1 \mathrm{~kg}$ MS de pradera tiene un costo relativo de 6 a 12 veces menos que producir $1 \mathrm{~kg}$ MS de concentrado (Valentine y Kemp, 2007). Esto hace que en estas regiones los sistemas pastoriles sean más rentables que los sistemas intensivos en confinamiento (Pulido et al., 2010). Uno de los factores más relevantes en la rentabilidad de los sistemas lecheros en base a 
praderas es la cantidad de pradera consumida por unidad de superficie, por lo que resulta importante conocer los factores que permiten maximizar este consumo (Balocchi et al., 2013).

Cuando se lleva a cabo el proceso de defoliación, factores como el aprendizaje, la disponibilidad y la selectividad harán que el pastoreo sea regulado por la intensidad, frecuencia y carga animal que se aplique sobre un área determinada (Valentine, 2000). En este sentido, criterios como la altura y disponibilidad de forraje se convierten en herramientas ampliamente utilizadas para el control del pastoreo mientras que el número de hojas será útil para determinar el estado de la planta y sus posteriores rebrotes (Balocchi y Ponce, 2001). A su vez, la forma en que los animales lleven a cabo el pastoreo influirá en la producción de fitomasa y en la heterogeneidad pratense por lo cual el pastoreo deber ser monitoreado adecuadamente para mantener la pradera en las condiciones que un productor necesita para su producción (López et al., 2003), las cuales dependerán de los objetivos productivos de éstos.

Cuando un animal comienza a consumir el forraje, factores como el estado fenológico, disponibilidad, concentración de nutrientes y palatabilidad, influyen en la digestión y consumo del mismo (Holmes, 1987; Hodgson, 1994) como también la habilidad que el animal tenga para su aprensión.

En praderas monofíticas, cuando la disponibilidad forrajera es alta, el animal comienza a seleccionar sobre la heterogeneidad existente, siendo las proporciones de la planta el factor fundamental para esa selección (Allen et al., 2011; Hodgson, 1986) dándose de manera gradual entre los componentes de la planta (Vavra y Ganskopp, 1998), siendo mayor el consumo en proporción de hojas verdes sobre los tallos y cuando se producen contrastes dentro de las plantas o en sus características físicas o químicas (Arnold, 1981). Mientras que en praderas polifíticas hay selección por las especies y luego los componentes de estas (Rutter, 2006).

Investigaciones sobre bovinos que son expuestos a praderas de la misma especie, han determinado que variables que afectan la selectividad en una pradera pueden ser la altura y disponibilidad de forraje (Bailey, 1995), atributos como el largo y ancho de la lámina (Smit et al., 2005), densidad de macollos (Forbes, 1982), y aspectos de calidad nutricional como digestibilidad, concentraciones de fibra y proteína cruda (Hodgson y Brookes, 1999), contenido de carbohidratos solubles (Moorby et al., 2006) y contenido de minerales (Horadagoda et al., 2009); entre los cuales se ha hecho referencia a la concentración de potasio (Morton et al., 2005; Leiber et al., 2009) y sodio en la pradera (Chiy y Phillips, 1991).

El potasio ha sido indicado como uno de los minerales que podría estar relacionado con un menor con- sumo de forrajes, cuando se encuentra en concentraciones bajas (Leiber et al., 2009) o elevadas (Morton et al., 2005). Estos últimos autores reportaron que el aumento del contenido de potasio por sobre un 4\% BMS de la pradera, disminuyó significativamente la tasa de bocado y tiempo de pastoreo de las vacas en el mes de octubre, no así en el mes de noviembre; adicionalmente este efecto ocurrió sólo en algunas localidades.

Las concentraciones de Ken praderas del sur de Chile están en un promedio de 3,15\% (Hernández, 2002). Éstas varían según la época del año (Roberts y Morton, 1999) con valores entre 1,8 a 5,0\% BMS, observándose las mayores concentraciones en otoño y primavera, mientras que las menores concentraciones ocurren en verano (Balocchi et al., 2001). Adicionalmente, la fertilización potásica es utilizada para incrementar el rendimiento y calidad nutritiva, y mejorar composición botánica de la pradera (Rodríguez y Domingo, 1987). No obstante, aplicaciones de fertilizantes potásicos aumentan las concentraciones de este mineral en la pradera, pudiendo gatillar una disiminución de otros cationes, especialmente de $\mathrm{Na}$ (Mosquera-Losada et al., 2004; Morton et al., 2004).

El sodio también ha sido relacionado con el consumo de forraje (Chiy y Phillips, 1991). Morton et al. (2005) observaron que aplicaciones de $\mathrm{NaCl}$ en praderas aumentaron significativamente el tiempo de pastoreo y la tasa de bocado sin haber alterado la calidad nutritiva de la pradera. Schneider et al. (1986) reportaron también un incremento en el tiempo de pastoreo y tasa de bocado cuando se suplementó a animales bajo estrés calórico con $\mathrm{NaCl}$.

Amaral et al. (1983) reportaron un incremento en la digestibilidad de la MS cuando es fertilizada con $\mathrm{NaCl}$. Existe evidencia de que los rumiantes seleccionarán una dieta con alto contenido de Na (Reid et al., 1966), incluso cuando se cumplen los requerimientos de $\mathrm{Na}$ (Michell y Moss, 1988). El consumo de MS voluntaria de alimentos conservados complementado con $\mathrm{NaCl}$ aumenta con hasta 5,5 $\mathrm{g} \mathrm{Na} \mathrm{kg}^{-1} \mathrm{MS}$ de la dieta (Schneider et al., 1986).

Adicionalmente, Chiy y Phillips (1991) demostraron que el sodio aplicado como fertilizante aumenta la proporción de forraje verde en praderas debido principalmente a cambios en la turgencia celular, mientras que Hemingway (1961) reportó que la aplicación de sodio como sal común en una pradera no tuvo efecto sobre el rendimiento. En este sentido, Cushnahan et al. (1995) plantean que la respuesta a la aplicación de $\mathrm{Na}$ en la pradera va a depender de las condiciones ambientales imperantes, como por ejemplo la humedad y concentración de $\mathrm{K}$ en el suelo. En los últimos años las posibilidades de deficiencia de este nutriente han aumentado a medida que se ha aplicado más $\mathrm{K}$ de fertilizante, lo que reduce el contenido de $\mathrm{Na}$ en el forraje (Balocchi et al., 2001; Morton et al., 2004); ya que exis- 
te una relación antagonista entre estos cationes, donde bajo condiciones de deficiencia de K, el Na puede ocupar su lugar (Hylton et al., 1967).

El objetivo de esta investigación es determinar el efecto que producen distintas relaciones de potasio/ sodio en una pradera permanente de Lolium perenne $\mathrm{L}$ sobre el consumo voluntario del animal.

\section{MATERIALES Y MÉTODOS}

\section{Localización y duración del estudio}

El estudio se realizó en la Estación Experimental Agropecuaria de la Universidad Austral de Chile (latitud sur $39^{\circ} 47^{\prime} 13^{\prime \prime}$ y longitud oeste $73^{\circ} 13^{\prime} 57^{\prime \prime}$ a 12 m.s.n.m) por un período de 7 meses (desde el 15 de marzo hasta el 15 de octubre del 2014).

El clima del sector se caracteriza por una temperatura promedio anual de $12,1^{\circ} \mathrm{C}$, con un valor medio mensual máximo de $16,9{ }^{\circ} \mathrm{C}$ en enero y un mínimo de $7,6^{\circ} \mathrm{C}$ en julio y una precipitación media anual de $2.500 \mathrm{~mm}$ (Balocchi et al., 2001).

El suelo en donde se llevó a cabo el estudio, es de origen Andisol (trumao), proveniente de cenizas volcánicas sobre depósitos marinos llamados cancagua, serie Valdivia, clasificado como Duric Hapludands con una capacidad de uso potencial de II y III (Nissen y Barría, 1976) y una pendiente de 1 a $8 \%$.

\section{Descripción del ensayo}

El ensayo se realizó sobre una pradera establecida de Lolium perenne L. (más de $90 \%$ de presencia de esta especie) de dos años de antigüedad, la cual era pastoreada por vacas lecheras. El sitio presentó niveles iniciales de $\mathrm{pH}\left(\mathrm{H}_{2} \mathrm{O}\right)$ de 6,0, P-Olsen $\left(\mathrm{mg} \mathrm{kg}^{-1}\right)$ de $17,8, \mathrm{~K}$ intercambiable $\left(\mathrm{mg} \mathrm{kg}^{-1}\right)$ 98, Na intercambiable (cmol+ $\mathrm{kg}^{-1}$ ) de 0,08 , Ca intercambiable $\left(\mathrm{cmol}+\mathrm{kg}^{-1}\right)$ de $5,03 \mathrm{y}$ $\mathrm{Mg}$ intercambiable $\left(\mathrm{cmol}+\mathrm{kg}^{-1}\right)$ de 0,37.

Al inicio del estudio, se realizó un corte de homogenización con una máquina segadora (Bertolini $140 \mathrm{~L}$, Reggio Emilia, Italia) a una altura de $5 \mathrm{~cm}$. Para lograr relaciones $\mathrm{K} / \mathrm{Na}$ contrastantes en la pradera se definieron dosis de 50 y $100 \mathrm{~kg} \mathrm{Na} \mathrm{ha}^{-1}$ (en forma de $\mathrm{NaCl}$ ), 400 y $800 \mathrm{~kg} \mathrm{~K}_{2} \mathrm{O} \mathrm{ha}^{-1}$ (en forma de $\mathrm{KCl}$ ), más un tratamiento que no recibió fertilización de Na y K. Las aplicaciones de fertilizante fueron realizadas al inicio del experimento y posterior a cada pastoreo. Cada tratamiento tuvo tres repeticiones distribuidas en bloques al azar en parcelas de $20 \times 10 \mathrm{~m}\left(200 \mathrm{~m}^{2}\right)$ cada una.

\section{Manejo del pastoreo y alimentación}

Las distintas praderas fueron pastoreadas de forma simultánea cuando éstas alcanzaron en promedio una fitomasa de 1.800-2.000 o 2.300-2.500 kg MS ha- ${ }^{-1}$ para invierno y otoño, y primavera respectivamente. De esta forma se llevaron a cabo tres pastoreos, los días 21 de mayo (P1), 20 de septiembre (P2) y 12 de octubre (P3). Para el pastoreo se utilizaron vacas en lactancia de raza Frisón Negro (PV: $526 \pm 49$ kg; PL: 19,7 $\pm 2,3$ kg ), las cuales fueron llevadas a las parcelas después de la ordeña de las 8:00 am para pastorear por un período de 6 horas o hasta dejar un residuo de $1.200-1.500 \mathrm{~kg}$ MS ha $^{-1}$ según la estación. Las vacas fueron suplmementadas con $4 \mathrm{~kg}$ TCO de concentrado comercial $\left(205 \mathrm{~g} \mathrm{~kg}^{-1}\right.$ PC; $363 \mathrm{~g} \mathrm{~kg}^{-1}$ FDN; $906 \mathrm{~g} \mathrm{~kg}^{-1} \mathrm{VD}$; ) durante las ordeñas y se les ofreció $6 \mathrm{~kg}$ MS de pradera permanente en la tarde de P1 y P3, mientras que en P2, estas recibieron $6 \mathrm{~kg}$ MS de ensilaje de pradera permanente. Adicionalmente recibieron $150 \mathrm{~g}$ diarios de sales minerales (100 g kg-1 Ca, $29 \mathrm{~g} \mathrm{~kg}^{-1} \mathrm{P}, 13 \mathrm{~g} \mathrm{~kg}^{-1} \mathrm{Mg}, 129,3 \mathrm{~g} \mathrm{~kg}^{-1}$ Na y $14 \mathrm{mg} \mathrm{kg}^{-1} \mathrm{~K}$ ). El número de vacas a pastorear se determinó a partir de la fitomasa disponible, fitomasa residual esperada, considerando una oferta de $6 \mathrm{~kg}$ de MS vaca ${ }^{-1}$ (sobre $5 \mathrm{~cm}$ de altura residual). De esta forma se utilizaron 28, 25 y 24 vacas para P1, P2 y P3 respectivamente. No existía delimitación entre parcelas de un mismo bloque, por lo que las vacas tenían libre acceso a cualquier tratamiento durante el evento de pastoreo. Cada bloque fue pastoreado en días distintos pero consecutivos por el mismo número de animales, es decir, el primer día pastorearon el bloque 1, segundo día el bloque 2 y el tercer día el bloque 3 . Al finalizar los tres días de pastoreo, se realizó un corte de homogenización con la segadora para tener una altura de pradera uniforme y procedió a aplicar las dosis de fertilizante asignadas a cada tratamiento.

\section{Variables evaluadas}

En este ensayo se evaluaron variables productivas y de composición de la pradera, además de consumo voluntario y tasa de defoliación por parte de los animales. La disponibilidad de MS fue estimada a partir de la altura comprimida con un plato medidor de forraje (Earle y McGowan, 1979), utilizando ecuaciones calibradas para cada época del año (Canseco et al., 2007). En cada parcela se realizaron 40 mediciones en forma de zig-zag previo y posterior a cada pastoreo. Después se realizó un corte de homogenización a una altura de $5 \mathrm{~cm}$ en todas las parcelas para medir el residuo generado. La cantidad de pradera removida fue determinada por la diferencia entre la fitomasa pre-pastoreo y la fitomasa residual por parcela

La altura de la pradera fue evaluada con una vara medidora (Sward Stick) en 40 puntos en forma de zigzag en cada parcela al inicio y final de cada pastoreo.

Para evaluar la dinámica de defoliación, se midió la altura comprimida y la altura no disturbada de cada parcela desde el inicio de pastoreo y durante las 6 horas siguientes con una frecuencia de 30 minutos. 


\section{Calidad nutritiva}

A las 15:00 horas el día anterior al pastoreo, se colectaron 10 muestras en cada parcela a $5 \mathrm{~cm}$ de altura y llevadas al laboratorio de Nutrición Animal de la Universidad Austral de Chile para determinación de MS según metodología de Bateman (1970). La composición nutricional fue determinada por espectroscopía de reflectacia el infrarrojo cercano (NIRS) con métodos de referencia para cenizas totales (CT), Proteína cruda (PC) por el método de micro-Kjeldhal según Bateman (1970), Proteína soluble (PS) por incubación en buffer y Micro Kjeldahl según Licitra et al. (1996), Energía Metabolizable (EM) según Garrido y Mann (1981), carbohidratos solubles (CHOS) según Maff (1985), Fibra detergente neutra (FDN) según Van Soest et al. (1991), Fibra detergente ácida (FDA). Los minerales Na y K se determinaron según las metodologías reportadas por AOAC (1996).

\section{Comportamiento en pastoreo}

El tiempo de las vacas pastoreando y echadas fue registrado visualmente y de manera continua por dos operarios. Cada 10 minutos y durante todo el evento de pastoreo, se registró el número de vacas que se encontraban pastoreando o echadas en cada parcela. El tiempo de pastoreo/echado fue calculado por la suma de todos los intervalos de 10 minutos en que los animales se encontraban realizando dicha actividad. La proporción de tiempo pastoreando fue estimada dividiendo el tiempo de pastoreo por el tiempo total que los animales permanecieron en la pradera (Perez-Ramirez et al., 2008).

\section{Diseño experimental y análisis estadístico.}

Se utilizó un diseño en bloques completos al azar con medidas repetidas en el tiempo, con 5 niveles de $\mathrm{K} / \mathrm{Na}$ en la pradera evaluados en tres pastoreos (medida repetida) para las variables Proteína Cruda, Proteína soluble, Cenizas totales, Energía Metabolizable, Fibra detergente neutro, Fibra detergente acido, Carbohidratos solubles, Potasio, Sodio, Relación K/Na, Fitomasa pre pastoreo (DisPre), Fitomasa post pastoreo (DisPos), Consumo aparente (Cap), Vacas pastoreando (VP), Vacas echadas (VE), Proporción vacas pastoreando (PrpP), Proporción vacas echadas (PrpE), Tiempo vacas pastoreando (TP) y Tiempo vacas echadas (TE).

Para las variables Fitomasa y Altura sin disturbar del ciclo de pastoreo, se utilizó un diseño de bloques completos al azar con con dos factores fijos (relación $\mathrm{K} / \mathrm{Na}$ en la pradera y pastoreo), con medidas repetidas en el tiempo (tiempo de pastoreo).

Los datos fueron sometidos a un análisis de varianza utilizando el procedimiento MIXED de SAS ${ }^{\circledR}$ (9.4). Las diferencias entre los tratamientos se determinaron cuando $\mathrm{P}<0,05$ utilizando la opción PDIFF con Tukey ajustado en SAS®.

\section{RESULTADOS Y DISCUSIÓN}

\section{Calidad nutritiva de la pradera y contenido de $\mathrm{K}$ y $\mathrm{Na}$.}

Las aplicaciones de Na y K no influyeron en la calidad nutritiva de la pradera (PC, PS, FDN, FDA, CHOS y EM) observándose una similar composición entre los tratamientos $(\mathrm{P}>0,05)$ a excepción de la concentración de cenizas totales, $\mathrm{K}$, Na y su relación $\mathrm{K} / \mathrm{Na}(\mathrm{P}<0,05)$. Si se observó una variación en el contenido de nutrientes, CT y $\mathrm{K}$ de la pradera de acuerdo al período de evaluación ( $\mathrm{P}<0,05$; Cuadro 1).

La calidad nutritiva de una pradera varia de acuerdo a las estaciones del año, a la fertilización y composición botánica (Keim et al., 2015). Los valores promedios obtenidos en todos los tratamientos están dentro del rango de referencia para una pradera permanente fertilizada en otoño en la provincia de Valdivia con excepción de la FDN (+5\%), la PS (-1\%), y las concentraciones de $\mathrm{K}$ y Na cuyos valores resultaron más altos que los normales debido a la fertilización usada en el estudio. Los valores reportados en el tratamiento testigo se encuentran dentro de los rangos normales de $\mathrm{K}(3.26 \%)$ y $\mathrm{Na}$ $(0.38 \%)$ para praderas del sur de Chile. El contenido de PC se encuentra dentro del rango de referencia señalado por Anrique et al. (2014) (20-25\%) pero menor al reportado por Pulido et al. (2010) (29,6\%). La EM promedio (2,75 Mcal kg-1 MS) fue menor a la señalada por Kolver y Muller (1998) (2,86 Mcal kg $\left.{ }^{-1} \mathrm{MS}\right)$ pero dentro del rango de referencia reportado por Parga (2003) (2,7-2,8 Mcal $\left.\mathrm{kg}^{-1} \mathrm{MS}\right)$ para una pradera de otoño. La FDN se encontró dentro del rango de referencia (40$55 \%$ ) indicado por Clark y Kanneganti (1998), y mayor al recomendado en dietas en vacas (25-35\%) por NRC (2001). Kolver y Muller (1998) señalan que la FDN no debe exceder de 35 a $40 \%$ para que no se afecte la velocidad de digestión y el tiempo de retención del forraje en el rumen que limita el consumo de MS.

La aplicación de 400 y $800 \mathrm{~kg} \mathrm{~K}_{2} \mathrm{O} \mathrm{ha}^{-1}$ aumentó las concentraciones de $\mathrm{K}$ en la pradera a valores promedio de 4,18 y 4,37\% BMS, mientras que para el tratamiento control y las aplicaciones de 50 y $100 \mathrm{~kg} \mathrm{Na} \mathrm{ha}^{-1}$ éstas estuvieron entre 3,07 - 3,26\% BMS. Adicionalmente, las concentraciones de $\mathrm{Na}$ en la pradera fueron mayores $(0,62-0,68 \%$ BMS $)$ con aplicaciones de 50 y $100 \mathrm{~kg} \mathrm{Na}$, mientras que la aplicación de 400 y $800 \mathrm{~kg}$ $\mathrm{K}_{2} \mathrm{O}$ generó una disminución de 0,15 y 0,23 unidades porcentuales respecto al control (0,38\% BMS). La aplicación de Ky Na modificó significativamente la relación $\mathrm{K} / \mathrm{Na}$ en la pradera $(\mathrm{P}<0,01)$, con valores tan extremos que fluctuaban desde 4,9-5,0 para aplicaciones de 50 y $100 \mathrm{~kg}$ Na hasta 23,1y 33,7 para 400 y $800 \mathrm{~kg} \mathrm{~K}$ O. Esto coincide con lo reportado por Hylton et al. (1967) 
Cuadro 1. Efecto de la fertilización con K o Na sobre la calidad nutritiva de la pradera durante las estaciones otoño - invierno - primavera.

Table 1. Effect of fertilization with $\mathrm{K}$ or Na on the nutritional quality of the pasture during autumn - winter - spring.

\begin{tabular}{|c|c|c|c|c|c|c|c|c|c|c|}
\hline & PC & PS & CT & FDN & FDA & CHOS & EM & $\mathbf{K}$ & $\mathrm{Na}$ & $\mathrm{K} / \mathrm{Na}$ \\
\hline & \multicolumn{6}{|c|}{ 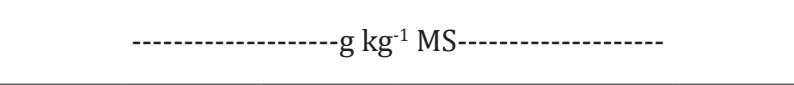 } & $\begin{array}{c}\text { Mcal } \\
\mathrm{kg}^{-1} \mathrm{MS}\end{array}$ & \multicolumn{2}{|c|}{------.\%-------- } & \\
\hline \multicolumn{11}{|l|}{ Fertilización } \\
\hline $0 \mathrm{~K}-50 \mathrm{Na}$ & 219 & 109 & $94^{\mathrm{c}}$ & 475 & 240 & 105 & 2,75 & $3,07^{\mathrm{b}}$ & $0,62^{\mathrm{a}}$ & $5,0^{\mathrm{d}}$ \\
\hline $0 \mathrm{~K}-100 \mathrm{Na}$ & 220 & 103 & $97^{\mathrm{b}}$ & 478 & 244 & 100 & 2,73 & $3,15^{b}$ & $0,68^{\mathrm{a}}$ & $4,9^{\mathrm{d}}$ \\
\hline $0 \mathrm{~K}-0 \mathrm{Na}$ & 215 & 102 & $94^{\mathrm{c}}$ & 479 & 239 & 102 & 2,74 & $3,26^{\mathrm{b}}$ & $0,38^{\mathrm{b}}$ & $8,6^{c}$ \\
\hline $400 \mathrm{~K}-0 \mathrm{Na}$ & 216 & 107 & $104^{a}$ & 474 & 240 & 107 & 2,74 & $4,18^{\mathrm{a}}$ & $0,22^{c}$ & $23,1^{\mathrm{b}}$ \\
\hline $800 \mathrm{~K}-0 \mathrm{Na}$ & 217 & 104 & $106^{a}$ & 483 & 241 & 108 & 2,73 & $4,37^{\mathrm{a}}$ & $0,15^{\mathrm{d}}$ & $33,7^{\mathrm{a}}$ \\
\hline Significancia & 0,91 & 0,918 & $<0,01$ & 0,619 & 0,766 & 0,106 & 0,830 & $<0,01$ & $<0,01$ & $<0,01$ \\
\hline \multicolumn{11}{|l|}{ Pastoreos } \\
\hline $\mathrm{P} 1$ & $253^{a}$ & $119^{a}$ & $106^{\mathrm{a}}$ & $495^{b}$ & $247^{b}$ & $84^{b}$ & $2,62^{c}$ & $3,81^{\mathrm{b}}$ & 0,45 & $10,1^{\mathrm{b}}$ \\
\hline $\mathrm{P} 2$ & $238^{\mathrm{b}}$ & $105^{b}$ & $100^{\mathrm{b}}$ & $540^{a}$ & $262^{a}$ & $90^{\mathrm{b}}$ & $2,77^{b}$ & $3,99^{\mathrm{a}}$ & 0,4 & $16,4^{\mathrm{a}}$ \\
\hline P3 & $161^{\mathrm{c}}$ & $91^{\mathrm{c}}$ & $92^{\mathrm{c}}$ & $400^{c}$ & $214^{\mathrm{c}}$ & $139^{a}$ & $2,83^{a}$ & $3,02^{c}$ & 0,37 & $18,7^{\mathrm{a}}$ \\
\hline Significancia & $<0,01$ & $<0,01$ & $<0,01$ & $<0,01$ & $<0,01$ & $<0,01$ & $<0,01$ & $<0,01$ & 0,117 & $<0,01$ \\
\hline \multicolumn{11}{|l|}{ Int. Fert*Past } \\
\hline Significancia & 0,08 & 0,913 & 0,860 & 0,365 & 0,967 & 0,028 & 0,813 & 0,394 & 0,493 & $<0,01$ \\
\hline
\end{tabular}

en donde se expresa que se puede presentar una interacción con los cationes hasta llegar a ser antagonista. Whitehead (2000) señala que al aplicar fertilizaciones altas en $\mathrm{K}$ sobre praderas la concentración de otros cationes como $\mathrm{Mg}$, Ca y Na disminuyen considerablemente, mientras que Balocchi et al. (2001) y MosqueraLosada et al. (2004) señalan que el efecto antagonista de las aplicaciones de $\mathrm{K}$ en la pradera afectan negativamente las concentraciones de $\mathrm{Na}$.

Adicionalmente, se observó una interacción significativa existente entre la aplicación de fertilizante y el pastoreo sobre la relación $\mathrm{K} / \mathrm{Na}(\mathrm{P}<0,01$; Figura 1). En esta se oberva que en el primer pastoreo las aplicaciones de $\mathrm{K}$ aumentaron la relación $\mathrm{K} / \mathrm{Na}$ a valores de entre 12 a 18, mientras que en el segundo y tercer pastoreo las relaciones aumentaron a valores de 25-35 y 31 - 48, respectivamente, lo que puede estar dado por acumulación de $\mathrm{K}$ en el suelo el cual es absorbido posteriormente por la planta, mientras que otra fracción es lixiviada (Morton et al., 2004).

\section{Efecto de las distintas relaciones $\mathrm{K} / \mathrm{Na}$ sobre la Fitomasa, consumo y comportamiento en pastoreo}

La aplicación de fertilizante potásico (KCl) o sódico $(\mathrm{NaCl})$ no afectaron la fitomasa diponibe de pradera $(\mathrm{P}>0,05)$. Este resultado difiere con Rodríguez y Do- mingo (1987) quienes observaron incremento en el rendimiento de fitomasa con aplicaciones creciente de potasio cuando éste es deficiente en el suelo, mientras que coincide con lo reportado por Hemingway (1961); Chiy y Phillips; (1999) Whitehead (2000), quienes encontraron efecto significativo sobre el rendimiento de fitomasa al aplicar $\mathrm{Na}$ en praderas dominadas por Lolium perenne (Cuadro 2). La fitomasa promedio prepastoreo (DisPre) fue muy similar en todos los tratamientos ( $>>0,05)$, con valores cercanos a las $2.400 \mathrm{~kg}$ MS ha-1, siendo rangos similares a los rendimientos de una pradera fertilizada dominada por Lolium perenne en la zona sur de Chile en los meses de mayo, septiembre, octubre (Araya et al., 2012).

La oferta forrajera está influida por la altura de la pradera (Kennedy et al., 2007) lo que afecta a su vez el consumo voluntario viéndose reflejado en una disminución cuando la altura es mayor (Taweel, 2006). Esto se debe a que las plantas se encuentran en estados más avanzados de desarrollo y por tanto, con menor calidad nutritiva como alimento (Nordheim-Vike y Volden, 2009). En esta investigación, se presentó una diferencia en la fitomasa pre y post pastoreo así como en el consumo aparente entre los pastoreos $(\mathrm{P}<0,01)$, lo que se puede argumentar por las diferentes tasas de crecimiento de la pradera en las distintas épocas del año. 


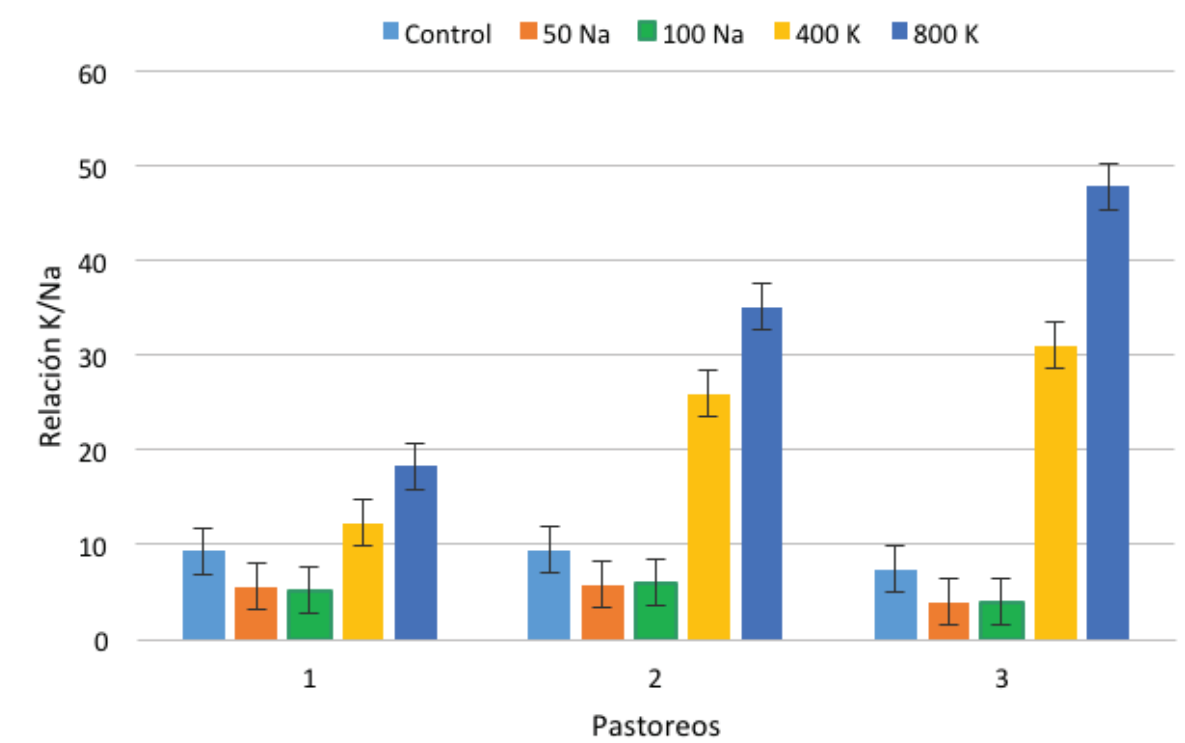

Figura 1. Interacción entre la fertilización con K o Na y el período de pastoreo sobre la relación K/Na en la pradera.

Figure 1. Interaction between $\mathrm{K}$ or $\mathrm{Na}$ fertilization rate and grazing period in the $\mathrm{K} / \mathrm{Na}$ ratio.

Cuadro 2. Efecto de la relación $\mathrm{K} / \mathrm{Na}$ sobre la fitomasa, consumo y comportamiento en pastoreo durante las estaciones otoño - invierno - primavera.

Table 2. Effect of $\mathrm{K} / \mathrm{Na}$ ratio on herbage mass, apparent intake and grazing behavior during autumn - winter - spring.

\begin{tabular}{|c|c|c|c|c|c|c|c|c|c|}
\hline & DisPre & DisPos & Cap & VP & VE & PrpP & PrpE & TP & TE \\
\hline & \multicolumn{3}{|c|}{ kg MS ha-1 } & \multicolumn{4}{|c|}{ No. Animales } & \multicolumn{2}{|c|}{ min vaca ${ }^{-1}$} \\
\hline \multicolumn{10}{|l|}{ Tratamiento } \\
\hline $0 \mathrm{~K}-50 \mathrm{Na}(\mathrm{K} / \mathrm{Na}: 4,9)$ & 2415 & 1457 & 958 & 3,1 & 2,2 & 0,59 & 0,40 & 231,1 & 162,2 \\
\hline $0 \mathrm{~K}-100 \mathrm{Na}(\mathrm{K} / \mathrm{Na}: 5,0)$ & 2447 & 1479 & 967 & 3,3 & 2,2 & 0,60 & 0,39 & 237,7 & 170,0 \\
\hline Testigo (K/Na: 8,6) & 2428 & 1434 & 994 & 2,7 & 1,8 & 0,61 & 0,38 & 224,4 & 156,6 \\
\hline $400 \mathrm{~K}-0 \mathrm{Na}(\mathrm{K} / \mathrm{Na}: 23,1)$ & 2454 & 1481 & 973 & 3,0 & 1,9 & 0,60 & 0,39 & 246,6 & 170,0 \\
\hline $800 \mathrm{~K}-0 \mathrm{Na}(\mathrm{K} / \mathrm{Na}: 33,7)$ & 2432 & 1498 & 933 & 3,1 & 1,9 & 0,62 & 0,37 & 237,7 & 157,7 \\
\hline Significancia & 0,971 & 0,874 & 0,925 & 0,533 & 0,878 & 0,991 & 0,991 & 0,488 & 0,958 \\
\hline \multicolumn{10}{|l|}{ Pastoreos } \\
\hline $\mathrm{P} 1$ & $2549^{a}$ & $1442^{\mathrm{b}}$ & $1106^{\mathrm{a}}$ & 3,2 & 2,3 & 0,58 & 0,41 & 239,3 & 173,3 \\
\hline $\mathrm{P} 2$ & $2491^{\mathrm{ab}}$ & $1399^{b}$ & $1091^{\mathrm{a}}$ & 3,2 & 1,7 & 0,66 & 0,33 & 241,3 & 150,6 \\
\hline P3 & $2266^{b}$ & $1567^{a}$ & $698^{\mathrm{b}}$ & 2,7 & 2,0 & 0,58 & 0,41 & 226,0 & 166,0 \\
\hline Significancia & $<0,01$ & $<0,01$ & $<0,01$ & 0,07 & 0,303 & 0,235 & 0,235 & 0,245 & 0,439 \\
\hline \multicolumn{10}{|l|}{ Int. Fert*Past } \\
\hline Significancia & 0,832 & 0,996 & 0,736 & 0,880 & 0,465 & 0,748 & 0,748 & 0,995 & 0,713 \\
\hline
\end{tabular}

DisPre: Fitomasa pre pastoreo; DisPos: Fitomasa post pastoreo; Cap: Consumo aparente; VP: Vacas pastoreando; VE: Vacas echadas; PrpP: Proporción vacas pastoreando; PrpE: Proporción vacas echadas; TP: Tiempo vacas pastoreando; TE: Tiempo vacas echadas. 
Las distintas relaciones $\mathrm{K} / \mathrm{Na}$ generadas no afectaron las variables relacionadas con consumo de pradera (DispPost y Cap) ni comportamiento en pastoreo (tiempo de pastoreo, número de animales pastoreando, proporción del tiempo en pastoreo) ( $\mathrm{P}>0,05$; Cuadro 2). No obstante, el consumo aparente fue mayor en los pastoreos 1 y 2 respecto al pastoreo 3 , en donde el consumo fue de $698 \mathrm{Kg}$ MS con una menor oferta forrajera (2.266 vs $2.549 \mathrm{Kg} \mathrm{MS}^{-1}$ ).

Cuando la disponibilidad es alta, los animales tienen la posibilidad de ser selectivos (Valentine, 2000) reflejándose un incremento en el número de bocados totales (Holmes et al., 2002) y una menor altura residual sin disturbar con una mayor profundidad de bocado (Tharmaraj et al., 2003). No obstante, esta mayor profundidad de bocado no se considera como un comportamiento de selectividad, ya que la mayor altura significaría solo una mayor accesibilidad a los macollos (Betteridge et al., 1994). Barrett et al. (2003) encontraron una relación directa entre el largo del macollo en pre pastoreo y la profundidad de bocado en cultivares de Lolium spp en estado vegetativo, por lo tanto a mayor altura, mayor profundidad de bocado, pero éste factor no puede ser tenido en cuenta en este estudio debido a que a los animales no se les dejó a libre selección sino fue definido por una oferta de pradera fija de MS de $6 \mathrm{~kg} \mathrm{vaca}^{-1}$.

Respecto a los patrones de comportamiento de pastoreo como el número de vacas pastoreando o echadas asi como el tiempo utilizado para llevar a cabo esas acciones, no presentaron diferencias estadísticas entre los tratamientos a pesar de haberse llevado a cabo metodologías desarrolladas por Shewmaker et al. (1997); Phillips et al. (1999) y Griffiths et al. (2003) en donde todos los animales tenían la misma probabilidad de pastorear los componentes pratenses sin ningún tipo de distorciones ambientales (López et al., 2003).

Respecto a la relación entre las concentraciones de $\mathrm{K}$ y Na en la pradera sobre el consumo y comportamiento en pastoreo, los resultados obtenidos en el presente estudio no concuerdan con Moselev (1980), Chiy y Phillips (1991), Chiy et al. (1993), Chiy y Phillips (1998), Phillips et al. (1999) quienes reportan un incremento en la palatabilidad cuando se aplicó fertilización con $\mathrm{Na}$ a la pradera (niveles alrededor de 0,2\% de $\mathrm{Na}$ en el tratamiento testigo). Por otro lado, Morton et al. (2005) reportaron que existe variabilidad en los resultados obtenidos en el consumo voluntario en praderas con diferentes niveles de $\mathrm{K}$, mostrándose mayor consumo en praderas bajas en potasio y una menor velocidad de bocado por vaca y tiempo de pastoreo cuando los niveles de potasio aumentaron de 2,54 a 3,46\%, sin embargo, este efecto no fue consistente en el tiempo ni entre distintas localidades. Edmeades y $\mathrm{O}^{\prime}$ Connor (2003) señalan que no hay respuesta animal a la fertilización o suplementación con $\mathrm{Na}$, cuando la concen- tración de en la pradera es mayor a un $0,10 \%$, lo que podría explicar la falta de respuesta de este ensayo, ya que los niveles del testigo sin sodio superaron ampliamente el $0,1 \%$ planteado por estos autores. Una de las limitantes del presente trabajo, obedece a caracer de la información respecto al consumo total de K, Na y Mg de los animales u otros indicadores como la relación $\mathrm{K} / \mathrm{Na}$ en la saliva, elementos que permitirían interpretar de mejor manera los resultados obtenidos.

\section{Efecto de la relación $\mathrm{K} / \mathrm{Na}$ en la pradera sobre la dinámica defoliación (kg MS ha-1) y reducción de altura (cm) en un ciclo de pastoreo.}

La medición de fitomasa disponible es una práctica dificultosa debido a la gran heterogeneidad existente dentro de la pradera a pesar de existir diferentes técnicas directas e indirectas para obtener el resultado (Teuber et al, 2007). Los métodos indirectos han logrado tener una valoración acertada en donde se ha identificado una alta correlación entre la altura sin disturbar y la comprimida (Earle y McGowan, 1979).

En el Cuadro 3 se oberva la fitomasa y altura sin disturbar durante el ciclo de pastoreo, como los efectos de las distintas relaciones $\mathrm{K} / \mathrm{Na}$, el pastoreo, tiempo de pastoreo y sus interacciones sobre la fitomasa y altura de la pradera. No se obervaron interacciones significativas sobre la altura de la pradera $(\mathrm{cm})$ o fitomasa de la pradera entre Fert*Pastoreo; Pastoreo*Fert; Fert*TP; Fert*Pastoreo*TP $(\mathrm{P}>0,05)$.

Al no observar diferencias entre las distintas relaciones K/Na sobre el consumo aparente o tiempo de pastoreo, se hacía relevante evaluar la reducción de fitomasa o altura de las praderas con distinta relación $\mathrm{K} / \mathrm{Na}$ durante el ciclo del pastoreo, y así determinar eventuales diferencias en preferencia. No obstante, como se señaló anteriormente no hubo interacción significativa entre la relación $\mathrm{K} / \mathrm{Na}$ en la pradera y el tiempo del pastoreo sobre la fitomasa y altura de la pradera, observándose un patrón de defoliación similar e independiente de la relación de $\mathrm{K} / \mathrm{Na}$ en la pradera (Figura 2). En general, la fitomasa inicial fue de $2.435 \mathrm{~kg} \mathrm{MS} \mathrm{ha}^{-1}$, disminuyendo cada media hora, hasta llegar a $1.430 \mathrm{~kg} \mathrm{MS} \mathrm{ha}^{-1}$ de residuo. La altura $(\mathrm{cm})$ de la pradera al inicio del pastoreo era de $17,7 \mathrm{~cm}$ decreciendo hasta llegar a $8,8 \mathrm{~cm}$ de residuo. En la primera hora posterior al ingreso de las vacas al potrero, el proceso de pastoreo es muy intenso y representa cerca del $60-70 \%$ del consumo total del evento de pastoreo. En las horas posteriores, la velocidad de consumo disminuye significativamente y se mantiene constante hasta el término del pastoreo, dinámica que coincide con la observada por (Chilibroste et al., 2012; Ribeiro et al., 2012; Pulido et al., 2015). Este fenómeno podría deberse a la capacidad ruminal de los animales, a una restricción ingestiva debido a la dificultad de cosecha por disminución en la altura de 
Cuadro 3. Efecto de la relación K/Na sobre la desaparición de MS y reducción de altura.

Table 3. Effect of K/Na ratio in the pasture on the DM and sward height disappearance.

\begin{tabular}{|c|c|c|}
\hline & $\begin{array}{l}\text { Fitomasa } \\
\text { Kg MS ha }^{-1}\end{array}$ & $\begin{array}{c}\text { Altura } \\
\mathrm{cm}\end{array}$ \\
\hline \multicolumn{3}{|c|}{ Fertilización-Relación $\mathrm{K} / \mathrm{Na}$} \\
\hline $0 \mathrm{~K}-50 \mathrm{Na}(\mathrm{K} / \mathrm{Na}: 4,9)$ & 1735 & 11,6 \\
\hline $0 \mathrm{~K}-100 \mathrm{Na}(\mathrm{K} / \mathrm{Na}: 5,0)$ & 1782 & 11,9 \\
\hline Testigo (K/Na: 8,6) & 1705 & 11,7 \\
\hline $400 \mathrm{~K}-0 \mathrm{Na}(\mathrm{K} / \mathrm{Na}: 23,1)$ & 1781 & 12,1 \\
\hline $800 \mathrm{~K}-0 \mathrm{Na}(\mathrm{K} / \mathrm{Na}: 33,7)$ & 1765 & 11,8 \\
\hline Significancia & 0,521 & 0,951 \\
\hline \multicolumn{3}{|l|}{ Pastoreos } \\
\hline P1 & $1800^{\mathrm{a}}$ & $10,8^{\mathrm{b}}$ \\
\hline $\mathrm{P} 2$ & $1673^{b}$ & $13,8^{\mathrm{a}}$ \\
\hline P3 & $1787^{\mathrm{a}}$ & $10,9^{b}$ \\
\hline Significancia & $<0.01$ & $<0.01$ \\
\hline \multicolumn{3}{|l|}{ Tiempo Pastoreo (TP) } \\
\hline $0 \mathrm{hr}$ & $2435^{a}$ & $17,8^{\mathrm{a}}$ \\
\hline $0,5 \mathrm{hr}$ & $1964^{\mathrm{b}}$ & $14,6^{\mathrm{b}}$ \\
\hline $1 \mathrm{hr}$ & $1783^{\mathrm{c}}$ & $12,7^{\mathrm{c}}$ \\
\hline $1,5 \mathrm{hr}$ & $1696^{\mathrm{d}}$ & $11,5^{\mathrm{d}}$ \\
\hline $2 \mathrm{hr}$ & $1648^{\mathrm{d}}$ & $10,5^{\mathrm{e}}$ \\
\hline $3 \mathrm{hr}$ & $1553^{\mathrm{e}}$ & $9,6^{f}$ \\
\hline $4 \mathrm{hr}$ & $1480^{\mathrm{f}}$ & $8,9^{g}$ \\
\hline $5 \mathrm{hr}$ & $1470^{\mathrm{f}}$ & $8,9^{\mathrm{g}}$ \\
\hline Significancia & $<0,01$ & $<0,01$ \\
\hline \multicolumn{3}{|l|}{ Int. Fert*Pastoreo } \\
\hline Significancia & 1,00 & 0,993 \\
\hline \multicolumn{3}{|l|}{ Int. Pastoreo*TP } \\
\hline Significancia & $<0,01$ & 0,912 \\
\hline \multicolumn{3}{|l|}{ Int. Fert*TP } \\
\hline Significancia & 0,999 & 0,999 \\
\hline \multicolumn{3}{|l|}{ Int. Trt*Pastoreo*TP } \\
\hline Significancia & 1,00 & 1,00 \\
\hline
\end{tabular}

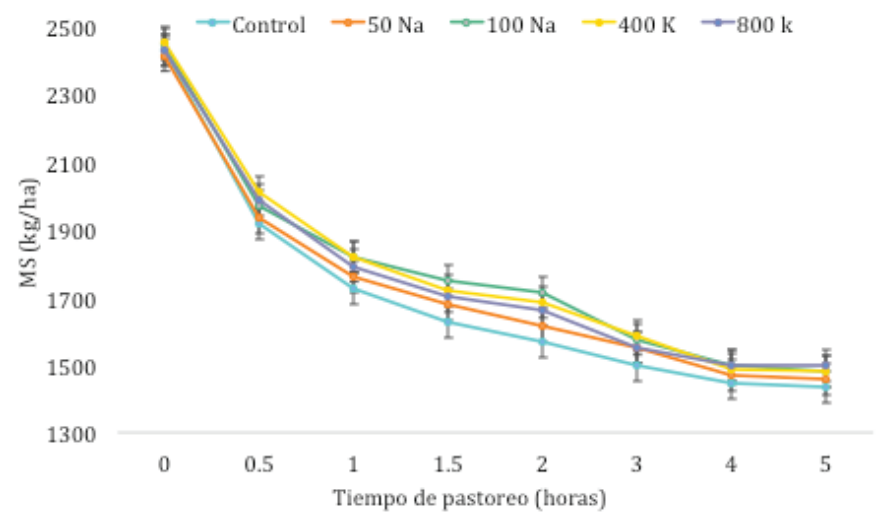

la pradera o bien, al cambio en la calidad del forraje disponible, expresado por las diferencias morfológicas que experimenta la pradera como posible consecuencia de la selectividad animal (Gregorini, 2012).

\section{CONCLUSIONES}

Las fertilizaciones con aplicaciones de potasio y sodio no incrementaron la fitomasa total, pero si aumentaron las concentraciones de $\mathrm{K}$ y $\mathrm{Na}$ en praderas permanentes de Lolium perenne en las estaciones de otoño-invierno-primavera. No obstante las contrastantes relaciones $\mathrm{K} / \mathrm{Na}$ en la pradera que se obtuvieron no se observó un efecto sobre el consumo voluntario de MS ni en el comportamiento ingestivo de vacas lecheras en pastoreo, las cuales concentran la actividad de pastoreo durante las primeras horas, sin manifestar preferencia frente a distintas relaciones $\mathrm{K} / \mathrm{Na}$.

\section{REFERENCIAS}

Allen, V. G., Batello, C., Berretta, E.J., Hodgson, J., Kothmann, M., Li, X., Mclvor, J., Milne, J., Morris, C., Peeters, A., Sanderson, M., The, F., Grazing Terminology. 2011. An international terminology for grazing lands and grazing animals. Grass and Forage Science 66, 2-28.

Amaral, D., Croom, W., Rakes, A., Leonard, E., Ljnnerud, A., 1983. Increased concentration of Sodium chloride on milk production of cows fed low fibre diets. Journal of Dairy Science 68, 2940-2947.

Anrique, R., Fuchslocher, R., Alfaro, M., Saldaña, R., 2014. Composición de alimentos para el ganado bovino (Cuarta Edición). Consorcio lechero y Universidad Austral de Chile, Valdivia.

Araya, R., Salazar, M., Contreras, K., Moscoso, C., Torres, A., Vásquez, M., Gaete, P., Wulf, M., Balocchi, O., Salidivia, E., Canalez, C. 2012. Manejo eficiente del pastoreo. Consorcio Lechero. Osorno, Chile. 51p.

Arnold, G., 1981. Grazing behavior, in: Morley, F. (Ed). Grazing Animals. Elsevier Scientific Publishing Company, Am-

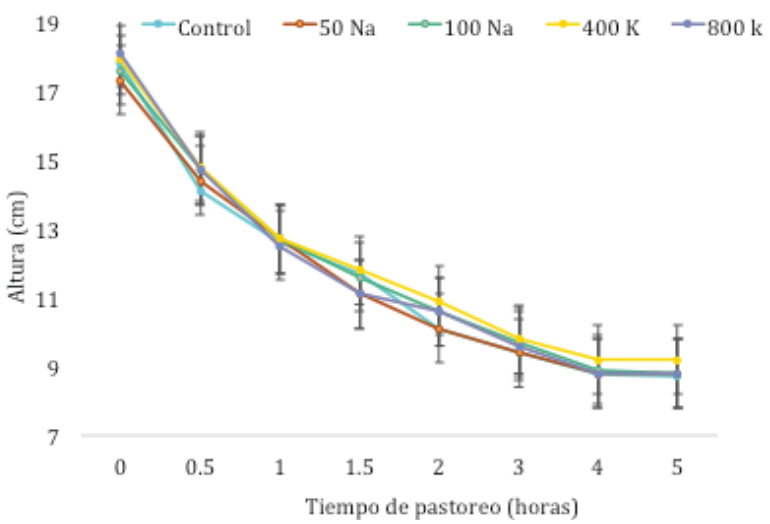

Figura 2. Desaparición de fitomasa (a) y altura (b) de la pradera en función de la fertilización con K o Na durante el ciclo de pastoreo.

Figure 2. Herbage mass (a) and sward height (b) disappearance throughout the grazing cycle as affected by Kor Na fertilizer applied 
sterdam, the Netherlands, pp. 79-104.

Bailey, D., 1995. Daily selection of feeding areas by cattle in homogeneous and heterogeneous environments. Applied animal behavior science 45, 183-200.

Barrett, P., Mc Gilloway, D., Laidlaw, A., Mayne, C., 2003. The effect of sward structure as influenced by ryegrass genotype on bite dimensions and short-term intake rate by dairy cows. Grass and Forage Science 58, 2-11.

Balocchi, O., Pinochet, D.T., Wittwer, F., Contreras, P., Echeverría, R., Guzmán, F., 2001. Rendimiento y composición mineral del forraje de una pradera permanente fertilizada con magnesio. Pesquisa Agropecuaria Brasileira $36(10), 1309-1317$.

Balocchi, O., Ponce, M., 2001. Manejo del pastoreo de vacas lecheras. Instituto deInvestigaciones Agropecuarias. Centro Regional de Investigación Remehue. Informativo No. 28, Osorno, 2p.

Balocchi L, O., Pulido, R. G., Merino, V. 2013., Oferta diaria de pradera: Efecto sobre el rendimiento y calidad de la pradera y sobre la producción de leche por vaca y por hectárea. Agro Sur 41(2), 9-16.

Betteridge, K., Fletcher, R., Liu, Y., Costall, D., Devantier, BP., 1994. Rate of removal of grass from mixed pastures by cattle, sheep and goat grazing. Journal of New Zealand grassland Association 56, 61-65.

Canseco, C., Demanet, R., Balocchi, O., Parga, J., Anwandter, V., abarzúa, A., Teuber, N., Lopetegui, J., 2007. Determinación de la disponibilidad de materia seca de praderas en pastoreo, in: Teuber, N., Balocchi, O., Parga, J. (Eds) Manejo de pastoreo. Proyecto FIA. Gobierno de Chile. Osorno, Chile, pp. 23-49.

Chilibroste, P., Mattiauda, D. A., Bentancur, O., Soca, P., Meikle, A., 2012. Effect of herbage allowance on grazing behavior and productive performance of early lactation primiparous Holstein cows. Animal Feed Science and Technology 173(3-4), 201-209.

Chiy, P., Phillips, C., 1991. The effects of sodium chloride application to pasture, or its direct supplementation, on dairy cow production and grazing preference. Grass and Forage Science 46, 325-331.

Chiy, P., Phillips, C., Omed, H., 1993. Sodium fertilizer application to pasture. Rumen dynamics. Grass and Forage Science 48, 249-259.

Chiy, P., Phillips, C., 1998. Sodium fertilizer application to pasture. Effects of combined applications with sulphur on herbage production and chemical composition in the season of application. Grass and Forage Science 53, 1-10.

Chiy, P., Phillips, C., 1999. Sodium fertilizer application to pasture. 8. Turnover and defoliation of leaf tissue. Grass and Forage Science 54, 297-311.

Clark, D., Kanneganti, P., 1998. Grazing management systems for dairy cattle, in: Cherney, P. (Eds). CABI, pp. 331.

Cushnahan, A., Bailey, J. S., Gordon, F. J., 1995. Some effects of sodium application on the yield and chemical-composition of pasture grown under differing conditions of potassium and moisture supply. Plant and Soil 176, 117-127.

Earle, D.F., McGowan, A.A., 1979. Evaluation and calibration of an automated rising plate meter for estimating dry matter yield of pasture. Australian Journal of Experimental Agriculture and Animal Husbandry, 19, 337-343.
Edmeades, D. C. \& O'Connor, M. B., 2003. Sodium requirements for temperate pastures in New Zealand: a review. New Zealand Journal of Agricultural Research 46, 37-47.

Forbes, T., 1982. Ingestive behaviour and diet selection in sheep and cattle. Ph. D. thesis. Edinburgh University, UK.

Gregorini, P., 2012. Diurnal grazing pattern: Its physiological basis and strategic management. Animal Production Science 52(7), 416-430.

Griffiths, W., Hodgson, J., Arnold, G., 2003. The influence of sward canopy structure on foraging decisions by grazing cattle. Grass and Forage Science 58, 112-124.

Hernández, 0., 2002. Variación estacional de la concentración de nutrientes minerales en cultivares de Lolium perenne L. Tesis de grado. Escuela de Agronomía. Universidad Austral de Chile. Valdivia, Chile.

Hemingway, R., 1961. Magnesium, potassium, sodium and calcium contents of herbage as influenced by fertilizer treatments over a three-year period. Journal of British Grassland Society 16, 106-116

Hodgson, J., 1986. Grazing behaviour and herbage intake, in: Frame, J. (Ed.). Grazing., London, England. British Grassland Society, pp. 51-64.

Hodgson, J., 1994. Manejo de pastos, teoría y práctica. México. Diana, $252 \mathrm{p}$.

Hodgson, J., Brookes, I., 1999. Nutrition of grazing animal, in: White, J. y Hodgson, J. (Eds.). New Zealand pasture and crop science. Oxford University, pp. 323-337.

Holmes, C., 1987. Pasture for dairy cattle, in: New Zealand Society of Animal Production. Livestock Feeding on Pasture, Occasional Publication No. 10, pp. 133-142.

Holmes, W., Brookes, I., Garrick, D., Mackenzie, D., Parkinson, T., Wilson, G., 2002. Milk production from pasture. Principles and practices. Massey University. Palmerston North, New Zealand, $602 \mathrm{p}$.

Horadagoda, A., Fulkerson, W. J., Nandra, K. S., Barchia, I. M., 2009. Grazing preferences by dairy cows for 14 forage species. Animal Production Science 49, 586-594.

Hylton, L., Ulrich, A., Cornelius, D., 1967. Potassium and sodium interrelations in growth and mineral content of Italian ryegrass. Agronomy Journal 59, 311-314.

Kennedy, E., O’Donovan, M., O’Mara, F., Murphy, J., Delaby, L., 2007. The effect of early lactation feeding strategy on the lactation performance of spring calving dairy cows. Journal of Dairy Science 90, 3060-3070.

Keim, J. P., López, I. F., Balocchi, O.A., 2015. Sward herbage accumulation and nutritive value as affected by pasture renovation strategy. Grass and Forage Science 70(2), 283-295.

Kolver, E., Muller, L., 1998. Performance and nutrient intake of high producing Holstein cows consuming pasture or a total mixed ration. . Journal of Dairy Science 81, 14031411.

Leiber, F., Wettstein, H.R., Kreuzer, M., 2009. Is the intrinsic potassium content of forages an important factor in intake regulation of dairy cows? Journal of Animal Physiology and Animal Nutrition 93(4), 391-399.

López, I., Hodgson, J., Hedderley, D., Valentine, I., Lambert, M., 2003. Selective defoliation by sheep according to slope and plant especies in the hill country of New Zealand. Grass and Forage Science 58, 339-349.

Moorby, J. M., Evans, R. T., Scollan, N. D., Macraet, J. C., Theodo- 
rou, M. K. 2006. Increased concentration of water-soluble carbohydrate in perennial ryegrass (Lolium perenne L.). Evaluation in dairy cows in early lactation. Grass and Forage Science 61, 52-59.

Morton, J. D., Roach, C. G., Tong, M. J., Roberts, A.H.C., 2004. Potassium in soil and pasture and leaching of cations on an allophanic soil in New Zealand. New Zealand Journal of Agricultural Research 47(2), 147-154.

Morton, J., Roach B., Roberts, H., 2005. Effect of potassium content and dusting of sodium chloride on the pasture preference of dairy cows. Grass and Forage Science 48, 29-37.

Moselev, G., 1980. Effects of variation in herbage sodium levels and salt supplementation on the nutritive value of perennial ryegrass for sheep. Grass and Forage Science 35, 105-113.

Michell, A., Moss, P., 1988. Salt appetites during pregnancy in sheep. Physiology \& Behavior 42, 491-493.

Mosquera-Losada, M. R., Gonzalez-Rodriquez, A., Rodriguez, A.R., 2004. Fertilization with nitrogen and potassium on pastures in temperate areas. Journal of Range Management 57(3), 280-290.

Nissen, J., Barria, J., 1976. Estudio agroecológico del predio Vista Alegre. Universidad Austral de Chile, Instituto de Suelos y Abonos. 30 p.

Nordheim-Viken, H., Volden. H., 2009. Effect of maturity stage, nitrogen fertilization and seasonal variation on ruminal degradation characteristics of neutral detergent fibre in timothy (Phleum pratense L.). Animal Feed Science and Technology 149(1-2), 30-59.

Perez-Ramirez, E., R. Delagarde, and L. Delaby., 2008. Herbage intake and behavioural adaptation of grazing dairy cows by restricting time at pasture under two feeding regimes. Animal 2(9):1384-1392.

Phillips, C., Youssef, M., Chiy, P., 1999. The effect of introducing timothy, cocksfoot and red fescue into a perennial ryegrass sward and the application of sodium fertilizer on the behavior of male and female cattle. Applied Animal Behaviour Science 61, 215-226.

Pulido, R., Muñoz, R., Jara, C., Balocchi, O., Smulders, J., Wittwer, F., Orellana, P., O'Donovan, M., 2010. The effect of pasture allowance and concentrate supplementation type on milk production performance and dry matter intake of autumn-calving dairy cows in early lactation. Livestock Science 132, 119-125.

Pulido, R. G., Ruiz-Albarrán, M., Balocchi, O.A., Nannig, P., Wittwer, F., 2015. Effect of timing of pasture allocation on production, behavior, rumen function, and metabolism of early lactating dairy cows during autumn. Livestock Science $177(0), 43-51$.

Reid, R., Jung, G., Murray, S., 1966. Nitrogen fertilization in re- lation to the palatability and nutritive value of orchard grass. Journal of Animal Science 25, 636-645.

Roberts, A., Morton, J., 1999. Fertilizer use on New Zealand dairy farms. Revised Edition. Auckland. New Zealand Fertilizer Manufacturers Association, $36 \mathrm{p}$.

Rodríguez, M., Domingo, M., 1987. Fertilización nitro-fosfopotásica en praderas naturales del País Vasco. Astos 17, 203-218.

Rutter, S.M., 2006. Diet preference for grass and legumes in free-ranging domestic sheep and cattle: Current theory and future application. Applied Animal Behaviour Science 97, 17-35.

Shewmaker, G., Mayland, H., Hansen, S., 1997. Cattle grazing preference among eight endophyte-free tall fescue cultivars. Agronomy Journal 89, 695-701.

Schneider, P., Beede, D., Wilcox, C., 1986. Responses of lactating cows lo dietary sodium source and quantity and potassium quantity during heat stress. Journal of Dairy Science 69, 99-110.

Smit, H., Tas, B., Taweel, H., Elgersma, A., 2005. Sward characteristics important for intake in six Lolium perenne varieties. Grass and Forage Science 60, 128-135.

Ribeiro, H. M. N., Peyraud, J. L., Delagarde, R., 2012. Foraging behavior and ruminal fermentation of dairy cows grazing ryegrass pasture alone or with white clover. Pesquisa Agropecuaria Brasileira 47(3), 458-465.

Taweel, H., 2006. Improving dry-matter intake of perennialryegrass pasture by dairy cows. Netherlands, pp. 159174.

Teuber, N., Balocchi, O., Parga, J., 2007. Manejo del pastoreo. Proyecto Fia. INIA Remehue, Universidad Austral de Chile, Universidad de la Frontera, Fundación para la innovación agraria. Osorno, Chile, pp. 114-134.

Tharmaraj, J., Wales, W., Chapman, D., Egan, A., 2003. Defoliation pattern, foraging behaviour and diet selection by lactating dairy cows in response to sward height and herbage allowance of a ryegrass-dominates pasture. Grass and Forage Science 58, 225-238.

Vavra, M., Ganskopp, D., 1998. Grazing behaviour in ungulates: current concepts and future challenges. Annals of Arid Zone 37, 319-335.

Valentine, J.F., 2000. Grazing Management (Second Edition). San Diego, USA. Academic Press. 659 p.

Valentine, I., Kemp, P., 2007. Pasture and supplement resources. In: Rattray, P.V., Brookes, I.M., Nicol, A.M. (Eds.), Pastures and Supplements for Grazing Animals: New Zealand Society of Animal production Occasional Publication No. 14., Hamilton NZ, pp. 3-11.

Whitehead, D., 2000. Nutrient elements in grassland. Soilplant-animal relationships. Wallingford, UK. CABI. 365$371 \mathrm{p}$. 